\title{
Um modelo estatístico para gestão de programas de pós-graduação
}

\section{A statistical model for post graduate programs management}

Paulo Sérgio Belchior Mesquita ${ }^{-}$- Universidade Estadual do Norte Fluminense - Centro de Ciência e Tecnologia Laboratório de Engenharia de Produção

Rodrigo Tavares Nogueira ${ }^{2}$ - Universidade Estadual do Norte Fluminense - Centro de Ciência e Tecnologia Laboratório de Engenharia de Produção

RESUMO O trabalho em questão tem como principal objetivo demonstrar a aplicabilidade da técnica de regressão logística, possibilitando estimar a probabilidade de obtenção de melhores notas e indicar as variáveis mais relevantes, para auxiliar os gestores dos programas de pós-graduação, na manutenção e melhoria dos conceitos CAPES em suas instituições.

Palavras-chave Regressão Logística. Avaliação CAPES.

ABSTRACT The research in question has as its main objective the demonstration of the applicability of the logistic regression technique, allowing the estimation of the probability of obtaining better grades and indicating the most relevant variables, to assist managers of post-graduate programs to maintain and improve the CAPES concepts in its institutions.

Keywords Logistic Regression. CAPES Evaluation. 


\title{
1. INTRODUÇÃO
}

Os programas de pós-graduação - Mestrado e Doutorado - são apreciados pela Fundação de Coordenação de Aperfeiçoamento de Pessoal de Ensino Superior (CAPES), que tem como meta prioritária, impulsionar o desenvolvimento nacional, através de um efetivo sistema nacional de avaliação.

Conforme Miranda e Almeida (2012), a importância de se ter um sistema de avaliação dos programas de pós-graduação no Brasil está relacionada a vários fatores. Dentre os mais relevantes, está a busca permanente da elevação dos padrões de qualidade desse nível de ensino, manter um sistema capaz de impulsionar a evolução de toda a pós-graduação mediante metas e desafios que expressem os avanços da ciência e tecnologia, fomentar estudos e atividades que, direta ou indiretamente, contribuam para o desenvolvimento e consolidação das instituições de ensino superior, manter intercâmbio e contato com outros órgãos, visando à celebração de convênios, acordos, contratos e manter um sistema de avaliação de cursos reconhecido e utilizado por outras instituições, capaz de apoiar o processo de desenvolvimento científico e tecnológico nacional.

Dois processos compõem a avaliação dos programas de pós-graduação: acompanhamento anual, e avaliação trienal de desempenho.

O acompanhamento anual é realizado no período compreendido entre os anos de realização das avaliações trienais. Tem por objetivo o estabelecimento de um diálogo entre a CAPES e as instituições de ensino superior promotoras de cursos de mestrado e doutorado com vistas à orientação da atuação dos programas de forma que possam elevar a qualidade de seu desempenho e superar os problemas que eventualmente estejam a enfrentar, se possível, antes da avaliação subsequente. $\mathrm{O}$ acompanhamento não implica a atribuição de conceitos aos programas, mas apenas a apresentação de um parecer com os comentários pertinentes.

\begin{abstract}
"Os resultados da avaliação trienal realizada pela CAPES, além de indicarem a qualidade do desempenho e a posição relativa de cada programa no contexto de sua respectiva área, servem de referência para as decisões dos órgãos governamentais de investimento na pesquisa e na pós-graduação e fundamentam as deliberações do Conselho Nacional de Educação sobre quais cursos de mestrado e de doutorado obterão, para vigência no triênio seguinte, a renovação de seu 'reconhecimento"'. (CAPES, 2014)
\end{abstract}

A avaliação, feita por comissões, é realizada ao final de cada triênio. As comissões avaliam o desempenho de cada programa a ela vinculado, atribuindo os conceitos Muito Bom (MB), Bom (B), Regular (R), Fraco (F) ou Deficiente (D) aos itens e respectivos quesitos da ficha de avaliação e uma nota inteira na escala 1 a 7 para cada programa, conforme organização da Tabela 1 . 
Tabela 1 - Quesitos e critérios de avaliação da CAPES.

\begin{tabular}{|c|c|}
\hline \multicolumn{2}{|r|}{ I-Proposta do programa } \\
\hline $\mathrm{x}_{1}$ & $\begin{array}{l}\text { Coerência, consistência, abrangência e atualização das áreas de concentração, linhas de pesquisa, projetos } \\
\text { e proposta curricular. }\end{array}$ \\
\hline $\mathrm{x}_{2}$ & $\begin{array}{l}\text { Planejamento do programa com vistas a seu desenvolvimento futuro, contemplando os desafios } \\
\text { internacionais da área na produção do conhecimento. }\end{array}$ \\
\hline$x_{3}$ & Infraestrutura para ensino, pesquisa e, se for o caso, extensão. \\
\hline \multicolumn{2}{|r|}{ II - Corpo docente } \\
\hline $\mathrm{x}_{4}$ & $\begin{array}{l}\text { Perfil do corpo docente, consideradas titulação, diversificação na origem da formação, aprimoramento e } \\
\text { experiência, e sua compatibilidade e adequação à proposta do programa. }\end{array}$ \\
\hline$x_{5}$ & $\begin{array}{l}\text { Adequação e dedicação dos docentes permanentes em relação às atividades de pesquisa e de formação do } \\
\text { programa. }\end{array}$ \\
\hline$x_{6}$ & Distribuição das atividades de pesquisa e de formação entre os docentes do programa. \\
\hline$x_{7}$ & $\begin{array}{l}\text { Contribuição dos docentes para atividades de ensino e/ou de pesquisa na graduação, com atenção tanto } \\
\text { à repercussão que este item pode ter na formação de futuros ingressantes na pó-graduação, quanto na } \\
\text { formação de profissionais mais capacitados no plano da graduação. }\end{array}$ \\
\hline \multicolumn{2}{|r|}{ III - Corpo discente, teses e dissertações } \\
\hline $\mathrm{x}_{8}$ & $\begin{array}{l}\text { Quantidade de teses e dissertações defendidas no período de avaliação, em relação ao corpo docente } \\
\text { permanente e à dimensão do corpo docente. }\end{array}$ \\
\hline$x_{9}$ & $\begin{array}{l}\text { Distribuição das orientações das teses e dissertações defendidas no período de avaliação em relação aos } \\
\text { docentes do programa. }\end{array}$ \\
\hline$x_{10}$ & $\begin{array}{l}\text { Qualidade das teses e dissertações e da produção de discentes autores da pós-graduação e da graduação } \\
\text { na produção científica do programa, aferida por publicações e outros indicadores pertinentes à área. }\end{array}$ \\
\hline$x_{11}$ & $\begin{array}{l}\text { Eficiência do programa na formação de mestres e doutores bolsistas: Tempo de formação de mestres e } \\
\text { doutores e percentual de bolsistas titulados. }\end{array}$ \\
\hline \multicolumn{2}{|r|}{ IV - Produção intelectual } \\
\hline$x_{12}$ & Publicações qualificadas do programa por docente permanente. \\
\hline$x_{13}$ & Distribuição de publicações qualificadas em relação ao corpo docente permanente do programa. \\
\hline $\mathrm{X}_{14}$ & Produção técnica, patentes e outras publicações consideradas relevantes. \\
\hline \multicolumn{2}{|r|}{ V- Inserção social } \\
\hline$x_{15}$ & Inserção e impacto regional e(ou) nacional do programa. \\
\hline$x_{16}$ & $\begin{array}{l}\text { Integração e cooperação com outros programas e centros de pesquisa e desenvolvimento profissional, } \\
\text { relacionados à área de conhecimento do programa, com vistas ao desenvolvimento da pesquisa e da pós- } \\
\text { graduação. }\end{array}$ \\
\hline$x_{17}$ & Visibilidade ou transparência dada pelo programa à sua atuação. \\
\hline
\end{tabular}

Fonte: Adaptado de CAPES (2014).

Os resultados da avaliação de cada programa são apresentados na "Ficha de Avaliação", que constam as notas e atributos dos vários quesitos e itens avaliados. 


\section{REFERENCIAL TEÓRICO}

A busca da fundamentação teórica desta pesquisa foi iniciada pela revisão do estado da arte, ou seja, das publicações mais recentes de artigos, livros, dissertações, teses e revisões sobre os seus temas centrais.

Pereira (2005) aplica as técnicas estatísticas multivariadas de análise discriminante e regressão logística para medir a influência das variáveis nos conceitos atribuídos pela CAPES aos programas de pós-graduação em administração, Neves e Costa (2006) propõem um método capaz de incorporar abordagens de diagnóstico estratégico (SWOT) e de tratamento de variáveis subjetivas (AMD) ao sistema de avaliação da CAPES; Leite et al. (2006) identificam os métodos quantitativos já aplicados à avaliação da CAPES; Oliveira e Freitas (2009) analisam o Modelo de Avaliação da CAPES e seus impactos sobre a vida profissional e pessoal dos professores de um programa de pós-graduação; Barros et al. (2012) investigam o suporte dado aos bolsistas de iniciação à pesquisa; Ensslin et al. (2006) exploram a autoavaliação de um programa de pós-graduação; Miranda e Almeida (2003) avaliam o uso de métodos de apoio à decisão multicritério para avaliação de programas de pós-graduação; Castro e Soares (2006) analisam a formulação, a avaliação e o aperfeiçoamento de instrumentos de avaliação dos programas de pós-graduação existentes no Brasil, com implicações para a alocação de recursos públicos; e Sguissardi (2006) responde a algumas questões acerca do "Modelo Capes de Avaliação".

\section{METODOLOGIA}

\subsection{Regressão Logística}

Conforme Cox e Snell (1989) e Hosmer e Lemeshow (2000), os modelos de regressão podem ser descritos como técnicas que permitem explicar a relação entre uma variável dependente, e um conjunto de variáveis independentes.

A regressão logística é uma técnica capaz de estimar a probabilidade de ocorrência de um determinado evento, a partir do estudo da relação das variáveis que provocam este evento.

Além de explicar relação entre as variáveis, possui algumas peculiaridades que em conjunto, faz com que a técnica se torne diferente dos demais modelos de regressão tradicionais.

\subsection{Modelo Estatístico}

Um modelo de regressão linear avalia o comportamento de uma variável dependente $(Y)$, em relação a uma ou mais variáveis independentes $\left(x_{i}\right)$ conforme as equações 1 e 2 abaixo.

Figura 1 - Equação 1.

$$
Y(x)=\alpha+\sum_{i=1}^{n}\left(\beta_{i} x_{i}+\varepsilon_{i}\right)
$$

Fonte: Elaborado pelos autores (2014). 
Figura 2 - Equação 2.

$$
E(Y \backslash x)=\alpha+\beta_{1} x_{1}+\beta_{2} x_{2}+\ldots+\beta_{n} x_{n}+\varepsilon_{n}
$$

Fonte: Elaborado pelos autores (2014).

Logo, neste modelo, é possível que a variável dependente assuma qualquer valor conforme a variação da variável independente, isto é, quando $x$ varia de menos infinito para mais infinito, $f(x)$ varia de menos infinito até mais infinito. Porém existem situações onde a variável dependente $(Y)$ é categórica e dicotômica, isto é, só pode ser enquadrada em duas categorias. Nestes casos, devido à simplicidade e relativa facilidade operacional, um dos modelos mais adequado para explicar o comportamento da variável dependente é o modelo logístico, dado pela equação 3 abaixo:

Figura 3 - Equação 3.

$$
f(z)=\frac{e^{z}}{1+e^{z}}=\frac{1}{1+e^{-z}}
$$

Fonte: Elaborado pelos autores (2014).

Este modelo continua fazendo uso de $n$ variáveis independentes para analisar o comportamento da variável dependente,

Figura 4 - Equação 4.

$$
\begin{array}{r}
z=\alpha+\beta_{1} x_{1}+\beta_{2} x_{2}+\cdots+\beta_{n} x_{n} \quad, \text { Substituindo, } \\
f(z)=\frac{1}{1+e^{-z}}=\frac{1}{1+e^{-\left(\alpha+\beta_{1} x_{1}+\beta_{2} x_{2}+\cdots+\beta_{n} x_{n}\right)}}=\frac{1}{1+e^{-\left(\alpha+\sum \beta_{i} x_{i}\right)}}
\end{array}
$$

Fonte: Elaborado pelos autores (2014).

Observe que na equação 4 quando $z$ varia de menos infinito para mais infinito, $f(z)$ varia de 0 a 1, apresentado um comportamento similar a trajetória de probabilidade. A expressão anterior $f(z)$ pode ser considerada como um conceito probabilistico. Então considere $n$ eventos independentes, em que $Y_{n}$ são variáveis independentes dicotômicas, consideradas como $n$ provas de Bernoulli, com probabilidade de sucesso $P(Y)$, isto é demonstrado na equação 5 abaixo:

Figura 5 - Equação 5.

$$
\begin{gathered}
P(Y)=\left\{\begin{array}{l}
1, \text { se sucesso } \\
0, \text { se fracasso }
\end{array}\right. \\
\text { Logo, } P\left(Y=1 \backslash x_{1}, x_{2}, \ldots, x_{n}\right)=\frac{1}{1+e^{-\left(\alpha+\sum \beta_{i} x_{i}\right)}}
\end{gathered}
$$

Fonte: Elaborado pelos autores (2014). 
Onde,

a) $P\left(Y=1 \backslash x_{1}, x_{2}, \ldots, x_{n}\right)$ : probabilidade condicional de ocorrer o evento $Y=1$ (variável dicotômica $\left.Y_{0,1}\right)$, dado vetor $X$

b) $x_{1}, x_{2}, \ldots, x_{n}$ : vetor $X$ (variáveis independentes);

c) $\alpha, \beta_{i}$ : parâmetros desconhecidos a serem estimados pelo método da maxima verossimilhança.

O odds é a medida de ocorrência que substitui a proporção quando esta não é aplicável. Ao tomar o exponencial de ambos os lados da equação de regressão, a equação pode ser reescrita como apresentada pela equação 6 abaixo:

Figura 6 - Equação 6.

$$
\text { odds }=\frac{p(\text { sucesso })}{p(\text { fracasso })}=\frac{p(\text { sucesso })}{1-p(\text { sucesso })}=e^{\beta_{0}} \cdot e^{\beta_{1} x_{1}} \ldots . e^{\beta_{k} x_{k}}
$$

Fonte: Elaborado pelos autores (2014).

Quando uma variável $x_{i}$ aumenta em 1 unidade, com todos os outros fatores permanecendo inalterados, então as chances vão aumentar por um fator $e^{\beta_{i}}$, como demonstra a equação 7 .

Figura 7 - Equação 7.

$$
e^{\beta_{i}\left(1+x_{i}\right)}-e^{\beta_{i} x_{i}}=e^{\beta_{i}\left(1+x_{i}\right)-\beta_{i x_{i}}}=e^{\beta_{i+} \beta_{i} x_{i}-\beta_{i} x_{i}}=e^{\beta_{i}}
$$

Fonte: Elaborado pelos autores (2014).

Este fator $e^{\beta_{i}}$ é a razão de chances (Odds ratio) para a variável independente $x_{i}$, e dá a relação das chances de aumento da variável de desfecho (Odds ratio $>1)$ ou redução $(<1)$, quando o valor da variável independente é aumentada por uma unidade.

A razão de chances (Odds ratio) é a razão entre as chances de um evento acontecer devido à influência de determinado fator, e a chance do evento acontecer sem a interferência deste fator, resumindo, é a medida de efeito que compara a probabilidade de determinada condição ocorrer entre grupos.

\subsection{Construção do Modelo}

Segundo a CAPES, na avaliação trienal 2013, referente ao período de 2010 a 2012, foram analisados 3.337 programas de pós-graduação, que compreendem 5.082 cursos, sendo 2.893 de mestrado, 1.792 de doutorado e 397 de mestrado profissional. O processo foi realizado durante o período de 30 de setembro de 2013 a 25 de outubro de 2013, quando cerca de 1.200 consultores estiveram reunidos na CAPES, distribuídos nas comissões de área de avaliação. 


\subsection{Tamanho da Amostra}

Não há um consenso entre os pesquisadores para o cálculo de tamanho da amostra numa regressão logística. Existe, porém, segundo Ribas (2011), uma regra de bolso para calculo de tamanho mínimo amostral, em que, é necessário pelo menos, casos para cada variável analisada, portanto, segundo esta regra, casos para esta pesquisa.

Segundo Bolfarine e Bussab (2005), a determinação do tamanho da amostra, com base numa estimativa conservadora da proporção populacional, é dada pela equação 8:

Figura 8 - Equação 8.

$$
\begin{gathered}
n=\frac{0,25 N\left(Z_{\frac{\alpha}{2}}\right)^{2}}{0,25 N\left(Z_{\frac{\alpha}{2}}\right)^{2}+(N-1) E^{2}} \cong 443 \\
\text { Onde, }\left\{\begin{array}{c}
n=\text { tamanho da amostra } \\
N=\text { tamanho da população }=3337 \text { programas } \\
E=\text { erro maximo da estimativa }=4 \% \\
Z_{\frac{\alpha}{2}}=\text { valor critico que corresponde a } 95 \% \text { de confiança }=1,96
\end{array}\right.
\end{gathered}
$$

Fonte: Elaborado pelos autores (2014).

Portanto, para esta pesquisa, de acordo com a população de 3337 programas de pós-graduação, o tamanho da amostra conservadora estimada para um nível de confiança $\alpha=95 \%$ e erro máximo de $4 \%$ é 443 cursos. Por uma questão de maior confiança, optou-se por inserir mais algumas observações na amostra, totalizando um total de 540 observações.

A base de dados utilizada neste trabalho está disponível em: https://www.dropbox.com/s/ xtfsqsbjef8hgal/Base\%20de\%20Dados\%20P\%C3\%B3s\%202014.xlsx?dl=0

\subsection{Codificação das variáveis}

Para construção da base de dados, foram utilizados os conceitos obtidos nos 17 quesitos (Tabela 1) e a nota de cada programa, disponibilizados pela CAPES nas fichas de avaliação. Codificados seguindo os critérios da Tabela 2, na codificação o valor "1" atribuído a nota da avaliação trienal 2013 corresponde ao acontecimento de interesse (sucesso), e o valor "0" (zero), ao seu complementar (fracasso).

O motivo das notas $\geq 4$ serem codificadas como sucesso, é que a CAPES considera a nota 3 , o padrão mínimo de qualidade para a recomendação do programa ao Conselho Nacional de Educação. Portanto uma "zona de risco". 
Tabela 2 - Critérios para codificação das variáveis.

\begin{tabular}{ccc}
\hline Se & For & Então \\
\hline \multirow{2}{*}{ Nota da avaliação $2013\left(y_{n}\right)$} & $\geq 4$ & 1 \\
\hline & $<4$ & 0 \\
\hline & Muito Bom "MB" \\
\cline { 2 - 3 } Item avaliado $\left(x_{i}\right)$ & Bom "B" & 5 \\
\cline { 2 - 3 } & Regular "R" & 3 \\
\cline { 2 - 3 } & Fraco "F" & 2 \\
\cline { 2 - 3 } & Deficiente "D" & 3 \\
\hline
\end{tabular}

Fonte: Elaborado pelos autores (2014).

\subsection{Estimação e Testes de Significância}

Após a construção da base de dados, foram estimados os parâmetros do modelo através do método da máxima verossimilhança que segundo Gujarati (2000) consiste em estimar os parâmetros desconhecidos de tal modo que a probabilidade de se observarem os dados é mais alta (ou máxima possível), e testados a significância dos coeficientes das variáveis independentes. Para a seleção das variáveis utilizou-se o método stepwise, que segundo Charnet et al. (2008) consiste em, após cada etapa de incorporação de uma variável, temos uma etapa em que uma das variáveis já selecionada pode ser descartada.

Tabela 3 - Ajuste do modelo geral.

\begin{tabular}{cc}
\hline \multicolumn{2}{c}{ Overall Model Fit } \\
\hline Null model -2Log Likelihood & 574,578 \\
\hline Full model -2Log Likelihood & 187,201 \\
\hline Chi-squared & 387,376 \\
\hline DF & 6 \\
\hline Significance level & $\mathrm{P}<0,0001$ \\
\hline
\end{tabular}

Fonte: Adaptado do R-project (2014).

Na Tabela 3 observa-se o modelo nulo -2Log Likelihood, que é obtido por $-2 \ln \left(\mathrm{L}_{0}\right)$ em que $\mathrm{L}_{0}$ é a probabilidade de se obter as observações, caso as variáveis independentes não tenham efeito sobre os resultados. Observa-se também o modelo completo - $2 \log$ Likelihood que é dado por $2 \ln (\mathrm{L})$ onde L é a probabilidade de se obter as observações com todas as variáveis independentes incorporadas no modelo. 
A diferença entre os resultados produz uma estatística chamada Qui-quadrado, que é uma medida do quanto as variáveis independentes afetam o resultado (variável dependente). Se o valor de $\mathrm{P}$ para o ajuste do modelo estatístico geral é menor que o convencional $(\leq 0,05)$, há evidências de que pelo menos uma das variáveis independentes contribui para a previsão dos resultados.

Tabela 4 - Coeficientes de regressão.

\begin{tabular}{cccc}
\hline \multicolumn{4}{c}{ Coefficients and Standard Errors } \\
\hline Variable & Coefficient & Std. Error & $\mathrm{P}$ \\
\hline $\mathrm{x}_{4}$ & 0,76123 & 0,28562 & 0,0077 \\
\hline $\mathrm{x}_{8}$ & 1,13703 & 0,23257 & $<0,0001$ \\
\hline $\mathrm{x}_{10}$ & 1,35086 & 0,23261 & $<0,0001$ \\
\hline $\mathrm{x}_{12}$ & 1,36281 & 0,26094 & $<0,0001$ \\
\hline $\mathrm{x}_{13}$ & 0,80715 & 0,22249 & 0,0003 \\
\hline $\mathrm{x}_{15}$ & 0,82099 & 0,28619 & 0,0041 \\
\hline Constant & $-23,6859$ & & \\
\hline
\end{tabular}

Fonte: Adaptado do R-project (2014).

Na Tabela 4 observam-se os coeficientes estatisticamente significantes da regressão. Uma variável independente, com um coeficiente de regressão não significante diferente de zero $(\mathrm{P}>$ $0,05)$ pode ser removido do modelo. Se a variável independente possui $\mathrm{P} \leq 0,05$, então ela contribui significativamente para a previsão da variável de desfecho (dependente).

O intervalo de confiança (Tabela 5) é usado para estimar a precisão do Odds ratio. Um grande intervalo indica baixo nível de precisão, enquanto um pequeno intervalo indica maior precisão.

Tabela 5 - Intervalo de confiança para odds ratio.

\begin{tabular}{ccc}
\hline \multicolumn{4}{c}{ Odds ratios and 95\% Confidence Intervals } \\
\hline Variable & Odds ratio & 95\% C.I. \\
\hline$x_{4}$ & 2,1409 & 1,2231 to 3,7474 \\
\hline$x_{8}$ & 3,1175 & 1,9762 to 4,9179 \\
\hline$x_{10}$ & 3,8607 & 2,4472 to 6,0907 \\
\hline$x_{12}$ & 3,9071 & 2,3428 to 6,5159 \\
\hline$x_{13}$ & 2,2415 & 1,4493 to 3,4668 \\
\hline$x_{15}$ & 2,2728 & 1,2970 to 3,9826 \\
\hline
\end{tabular}

Fonte: Adaptado do R-project (2014). 
Conforme Corrar et al. (2011) o teste Hosmer e Lemeshow (Tabela 6) verifica se existem diferenças significativas entre as classificações realizadas pelo modelo e a realidade observada (Tabela 7).

Tabela 6 - Teste de Hosmer \& Lemeshow.

\begin{tabular}{cc}
\hline \multicolumn{3}{c}{ Hosmer \& Lemeshow test } \\
\hline Chi-squared & 6,4298 \\
\hline DF & 7 \\
\hline Significance level & $P=0,4906$ \\
\hline
\end{tabular}

Fonte: Adaptado do R-project (2014).

O teste de Hosmer e Lemeshow indica que os valores preditos não são significativamente diferentes dos observados $(\mathrm{P}>0,05)$.

A Tabela de classificação a seguir emitida pelo R-project, exibe a taxa de acerto obtida, quando são utilizadas as variáveis independentes selecionadas (Tabela 5). Em um modelo perfeito, todos os casos estariam na diagonal e a porcentagem total correta seria de 100\%. Na Tabela 7 é possível observar as seguintes informações:

a) Sensibilidade $(95,9 \%)$ - mede a capacidade do modelo em classificar corretamente os programas com nota maior ou igual a quatro $(\geq 4)$, dado que ele realmente possui nota maior ou igual a quatro $(\geq 4)$;

b) Especificidade (80,2\%) - mede a capacidade do modelo em classificar corretamente os programas com nota menor que quatro $(<4)$, dado que ele realmente possui nota menor que quatro $(<4)$. Um bom modelo possui um alto valor para a sensibilidade e para a especificidade, pois ele classificará corretamente aqueles que podem obter nota $\geq 4$ (sucesso) e nota $<4$ (fracasso);

c) Em média obtém-se 92,4\% de acurácia (grau de exatidão ou precisão demonstrado pelo modelo) nas predições.

Tabela 7 - Tabela de classificação.

\begin{tabular}{cccc}
\hline \multicolumn{2}{c}{ table (cut-off value $\mathrm{p}=0,5)$} & \\
\hline \multicolumn{2}{c}{ Numero de casos } & percentagem (\%) \\
\hline observados & previstos & \\
\hline Fracasso & 28 & 24 & 80,2 \\
\hline Sucesso & 404 & 402 & 95,9 \\
\hline Porcentagem total & & & 92,4 \\
\hline
\end{tabular}

Fonte: Adaptado do R-project (2014). 


\section{RESULTADOS}

Após a estimação dos parâmetros e os testes de significância, são feitas algumas considerações:

a) Das dezessete variáveis analisadas, seis foram consideradas como sendo as mais significativas, portanto que melhor explicam as notas obtidas pelos programas de pós-graduação.

b) Todas as seis variáveis selecionadas exercem impacto positivo para aumentar a nota do programa.

c) A variável que exerce maior impacto é a $x_{12}$ (publicações qualificadas do programa por docente permanente).

Substituindo-se os coeficientes e as variáveis selecionadas na forma geral do modelo regressão logística, obtém-se o modelo ajustado demonstrado na equação 9 a seguir:

Figura 9 - Equação 9.

$$
\begin{aligned}
& P(Y=1)=P(\text { Sucesso })=P(\text { Nota } \geq 4)=\frac{1}{1+e^{-\left(\beta_{0}+\sum \beta_{i} x_{i}\right)}}
\end{aligned}
$$

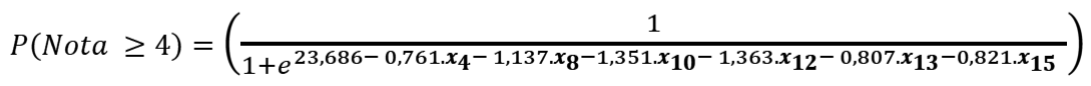

Fonte: Elaborado pelos autores (2014).

Pode-se observar através do Odds ratio (Tabela 5) que para cada mudança de 1 unidade no conceito da variável (perfil do corpo docente) as chances de obter uma nota maior ou igual a quatro são 2,15 maiores, já para a variável (Quantidade de teses e dissertações defendidas no período de avaliação) são 3,11 maiores, e assim por diante.

Com o modelo de regressão logística obtido é possível responder a questões importantes para a gestão dos programas de pós-graduação, tais como:

a) Quais os itens mais relevantes, ou que mais influenciam na nota final da avaliação CAPES?

- Observa-se que das seis variáveis selecionadas para o modelo, as três que causam maior impacto na nota final da avaliação CAPES são relacionadas com a produção científica do corpo docente e discente: Publicações qualificadas do programa por docente permanente), (Qualidade das teses e dissertações e da produção de discentes autores da pós-graduação e da graduação na produção científica do programa), (Quantidade de teses e dissertações defendidas no período de avaliação, em relação ao corpo docente permanente e à dimensão do corpo docente. Indicando, portanto, que os programas de pós-graduação devem investir na quantidade e qualidade das publicações de seus docentes e discentes. 
b) Qual a probabilidade de obter melhor nota na próxima avaliação CAPES, caso sejam mantidas os mesmos conceitos nos itens de avaliação?

- A substituição dos conceitos CAPES atuais no modelo ajustado, fornece a probabilidade de um programa de pós-graduação atingir uma nota maior ou igual a quatro na próxima avaliação, desde que sejam mantidas as mesmas notas das variáveis não utilizadas no modelo. Por exemplo, um programa que obteve conceito fraco em todas as variáveis do modelo na ultima avaliação, tem probabilidade de aproximadamente de conseguir uma nota na próxima avaliação trienal CAPES.

Lembrando que a codificação para o conceito FRACO é igual a (Tabela 2), temos a equação 10 a seguir:

Figura 10 - Equação 10.

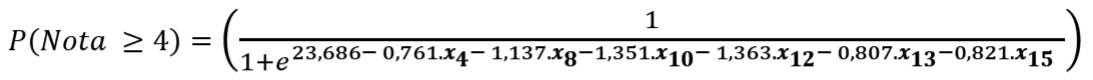

$$
\begin{aligned}
& P(\text { Nota } \geq 4)=\left(\frac{1}{1+e^{23,686-0,761(2)-1,137(2)-1,351(2)-1,363(2)-0,807(2)-0,821(2)}}\right) \cong 8,19 \%
\end{aligned}
$$

Fonte: Elaborado pelos autores (2014).

c) E se os conceitos desses itens fossem alterados, qual seria a probabilidade de obter melhor nota na próxima avaliação CAPES?

- Esta é uma situação interessante, pois permite fazer simulação usando combinação de conceitos, possibilitando assim saber de antemão quais notas devem ser alcançadas para atingir um conceito maior ou igual ao conceito atual, possibilitando que os programas dirijam os investimentos para determinados itens pré-selecionados.

d) Quais seriam os conceitos necessários para o programa ser incluído no grupo de sucesso $($ nota $\geq 4)$ ?

- Neste caso, conforme (CORRAR; PAULO; FILHO, 2011), resultados superiores a são assumidos com possível sucesso, e inferiores como possível fracasso. Portanto a simulação de resultados que obter probabilidade de sucesso de $50 \%$ será a combinação mínima para que o programa alcance uma nota maior ou igual a quatro na próxima avaliação CAPES.

\section{DISCUSSÕES}

Ao analisar modelo de regressão logística ajustado, nota-se claramente a importância da produção acadêmica cientifica nas avaliações de programas de pós-graduação realizada pela CAPES.

Observa-se também que a quantidade de publicações tem mais influência na nota de final da avaliação, que a qualidade das publicações, sendo este ponto, assunto que gera polêmica e alvo de critica entre diversos pesquisadores da área acadêmica.

Apesar da polêmica quantidade $\mathrm{X}$ qualidade não ser o objeto de estudo deste trabalho, acredita-se ser este, assunto importante, merecendo futuras discussões. 


\section{CONCLUSÕES}

Neste artigo, buscou-se apresentar aos gestores de cursos de pós-graduação, uma metodologia capaz de contribuir na busca por melhores notas em avaliações futuras da CAPES.

Por meio do modelo de regressão logística, foi possível identificar os itens que mais contribuem para a melhoria das notas dos programas de pós-graduação avaliados pela CAPES.

Observou-se que todas as variáveis selecionadas para o modelo, contribuem para aumentar a probabilidade de obtenção de melhores avaliações, e a ordem de "influência" das variáveis é: $x_{12}>$ $x_{10}>x_{8}>x_{15}>x_{13}>x_{4}$, onde $x_{12}$ (Publicações qualificadas do programa por docente permanente), $x_{10}$ (Qualidade das teses e dissertações e da produção de discentes autores da pós-graduação e da graduação), $x_{8}$ (Quantidade de teses e dissertações defendidas no período de avaliação), $x_{15}$ (Inserção e impacto regional e(ou) nacional do programa), $x_{13}$ (Distribuição de publicações qualificadas em relação ao corpo docente permanente do programa), e $x_{4}$ (Perfil do corpo docente).

Os resultados verificados, mostram que a variável que mais influencia na melhoria do conceito CAPES, é a que diz respeito a publicações qualificadas do programa por docente permanente. Este indicador é representado majoritariamente por artigos publicados em periódicos classificados nos estratos superiores do Qualis da área, indicando a importância das publicações para a avalição da CAPES.

A partir do modelo ajustado é possível que os gestores façam simulação da probabilidade de obtenção de melhores notas em seus respectivos programas, com a finalidade de obter informações para otimizar recursos em suas instituições.

Os fundamentos teóricos e resultados apresentados neste trabalho apoiam a utilização da regressão logística para estimar a probabilidade de um programa de pós-graduação obter melhores notas na avaliação trienal da CAPES.

\section{REFERÊNCIAS}

BARROS, V. F. A.; SOUZA, M. A. R.; MACHADO, S. S. Portal de periódicos da CAPES: a importância da investigação científica na iniciação científica. Cadernos de educação, tecnologia e sociedade, v. 3, n. 1, p. 47-53, 2012.

BOLFARINE, H.; BUSSAB, W. O. Elementos de amostragem. São Paulo: Editora Blucher, 2005.

CAPES - FUNDAÇÃO DE COORDENAÇÃO DE APERFEIÇOAMENTO DE PESSOAL DE NÍVEL SUPERIOR. Caracterização do Sistema de Avaliação da Pós-Graduação. Disponível em: $<$ http://www.capes.gov.br/conselho-superior/91-conteudo-estatico/avaliacao-capes/6871-caracterizacao-do-sistema-de-avaliacao-da-pos-graduacao $>$. Acesso em: nov. 2014.

CASTRO, C. M.; SOARES, G. A. D. Avaliando as avaliações da CAPES. Revista de Administração de Empresas, v. 23, n. 3, p. 63-73, 2006.

CHARNET, R.; FREIRE, C. A. L.; CHARNET, E. M. R.; BONVINO, H. Análise de modelos de regressão linear. Campinas, SP: Editora da Unicamp, 2008.

CORRAR, L. J.; PAULO, E.; FILHO, J. M. D. Análise Multivariada: para os cursos de administração, ciências contábeis e economia. FIPECAPI - Fundação Instituto de pesquisas Contábeis, Atuariais e Financeiras. São Paulo: Atlas, 2011. 
COX, D. R.; SNELL, E. J. Analysis of Binary Data. 2nd ed, London: Chapman \& Hall, 1989.

ENSSLIN, S. R.; IGARASHI, D. C. C.; ENSSLIN, L.; PLADINI, E. P. Construção de um modelo híbrido para auto-avaliação de um programa de pós-graduação - a fase de estruturação. In: Simpósio de Engenharia de Produção, 8, 2006, São Paulo. Anais... São Paulo: SIMPEP, 2006.

GUJARATI, D. N. Econometria básica. São Paulo: Pearson Makron Books, 2000.

HOSMER, D. W.; LEMESHOW, S. Applied Logistic Regression. New York: John Wiley, 2000.

LEITE, M. F. B.; VIANA, A. B. N. Métodos quantitativos na avaliação da CAPES: uma pesquisa bibliográfica. FACEF Pesquisa, v. 9, n. 2. 2006.

MIRANDA, C. M. G.; ALMEIDA, A. T. Avaliação de pós-graduação com método ELECTE TRI - o caso de Engenharias III da CAPES. Revista Produção, v. 3, n. 3, 2003.

NEVES, R. B.; COSTA, G. C. Avaliação dos programas de pós-graduação: proposta baseada na integração ELECTRE TRI, SWOT e sistemas CAPES. Sistemas \& Gestão, v. 1, n. 3, p. 276-298, 2006.

OLIVEIRA, M. A. M.; FREITAS, M. V. M. T. F. O atual modelo de avaliação da CAPES: seus impactos sobre as vidas profissional e pessoal dos docentes de um programa de pós-graduação em letras. Educação em foco, v. 12, n. 13, p. 29-52, 2009.

PEREIRA, G. G. Avaliação da Capes: abordagem quantitativa multivariada dos programas de administração. 2005. Dissertação (mestrado em Administração) - Faculdade de Economia, Administração e Contabilidade, Universidade de São Paulo, São Paulo. 2005. Disponível em: <http:// www.teses.usp.br/teses/disponiveis/12/12139/tde-25092007-004335/>. Acesso em: 07 jan. 2015.

R CORE TEAM. R: A language and environment for statistical computing. $\mathbf{R}$ Foundation for Statistical Computing, Vienna, Austria. Disponível em: <http://www.R-project.org $>$. Acesso em: 2014.

RIBAS, J. R.; VIEIRA, P. R. C. Análise Multivariada com o uso do SPSS. Rio de Janeiro: Editora Ciência Moderna Ltda, 2011.

SGUISSARDI, V. A avaliação defensiva no "modelo CAPES de avaliação" - é possível conciliar avaliação educativa com processos de regulação e controle do Estado? Perspectiva, v. 24, n. 1, p. 49-88, 2006. 